\title{
A bet on quantum
}

\section{The UK is investing ambitiously in quantum technologies.}

In December 2013, the Chancellor of the Exchequer, George Osborne, announced that the UK would fund a $£ 270$ million initiative to establish a National Quantum Technologies Programme to convert quantum physics research into commercial products ${ }^{1}$. Following almost a year of consultations, proposals and evaluations, the government has now revealed how the biggest chunk of this investment (roughly $£ 120$ million) will be allocated across a national network of Quantum Technology Hubs that will be led by the universities of Birmingham, Glasgow, Oxford and York, but will also involve another 13 universities and 132 companies.

The four hubs were selected from a shortlist of eight proposals, and will each focus on different applications spanning quantum sensing and metrology, quantum communications, and quantum computing and simulation. Moreover, an additional $£ 4$ million has been set aside to establish a Quantum Metrology Institute at the National Physical Laboratory (NPL), the UK's measurement standards laboratory.

The UK has a strong base in fundamental quantum research, so the rationale to develop its applications and encourage their exploitation in industry is sound. However, an inability to follow up and successfully exploit its research for commercialization is arguably a weakness that has historically plagued Britain. Why should it be any different this time?

Although it is inevitable that any flagship investment focusing on a particular area of research will lead to some disappointment from scientists working on 'excluded' areas, there are grounds to be optimistic. For one thing, there is an emphasis on playing to existing strengths and working towards technologies that can realistically be delivered on the timescale of years. Quantum metrology is one such example: atomic clocks have been established for decades, but there are plans for the NPL to use them as a means to provide a centralized time with which to synchronize high-frequency financial transactions in the City of London. In effect, the NPL would be providing accurate time, which would help to prevent traders from exploiting tiny time differences between the networks of different banks for profit.

\section{There is an emphasis on playing to existing strengths and working towards technologies that can realistically be delivered on the timescale of years.}

The deployment of so-called second generation quantum technologies, namely those harnessing effects such as quantum superposition and entanglement ${ }^{2}$, is a little further off. One area that has received much coverage and attention due to its immense potential is that of quantum information processing ${ }^{3}$. Alas, solid-state quantum systems - widely believed to be the most likely means for realizing quantum computers - are notably absent from the main missions of the four quantum hubs. This decision is most likely down to cold, hard pragmatism: other areas such as quantum communications, which includes quantum cryptography, appear to be more mature, although they still face formidable challenges if they are to become widespread technologies.

On the face of it, the National Quantum Technologies Programme strikes a reasonable balance between scientific ambition and likely technological payoff. Moreover, the quantum hubs themselves are conceived to operate as part of a network that is designed to be inclusive of the wider research and industry community - they are not designed to operate in isolation. Research has a knack for generating the unexpected, and this structure should provide an ability to respond to changes in the research landscape efficiently.

Perhaps most importantly, by integrating themselves within the British university system, the hubs will play a central role in creating a generation of world-class researchers. The seeds of a new school of thought are being sown, they must now be nurtured.

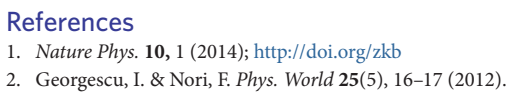

\section{Lowering the blinds}

\section{Nature Physics will soon offer the option of double-blind peer review, for which authors and referees are anonymous.}

In 1967 Nature adopted 'single-blind' peer review to evaluate manuscripts a model that hides the identities of the reviewers, who often feel more at liberty to speak honestly and critically this way. Last year, Nature Geoscience and Nature Climate Change started trials of double-blind peer review, where the authors' identities are also hidden, and several journals now offer this facility. Nature Physics will be next.
Peer review is not perfect. Publishers are always thinking of ways to improve this process, and author anonymity may redress any perceived biases against gender, race, age or geographical location. Or perhaps not. During the trials, surveyed authors generally agreed that double-blind peer review was a good idea in principle, but ultimately, take-up was low, peaking at $20 \%$ for the two journals (Nature Nano. 9, 871-872; 2014).

Such short trials are inconclusive, but the impression is that double-blind peer review is a viable option, which is why all the Nature journals will offer it as an alternative from next week. Authors will have to opt in and prepare their manuscripts accordingly.

In physics, the popularity of the preprint archive would seem at odds with a doubleblind peer review system, and authors may wish to speak about upcoming work at conferences. Ultimately, the choice will be up to the authors. 